SCIENTIFIC CORRESPONDENCE

\title{
Patchy atrophy and lacquer cracks predispose to the development of choroidal neovascularisation in pathological myopia
}

\author{
K Ohno-Matsui, T Yoshida, S Futagami, K Yasuzumi, N Shimada, A Kojima, T Tokoro, \\ M Mochizuki
}

\begin{abstract}
Aims: To determine the incidence and predisposing findings for choroidal neovascularisation (CNV) in a large series of highly myopic patients.

Methods: The medical records of 218 consecutive patients (325 eyes) with myopic fundus changes in the macula were reviewed. The incidence of CNV during a follow up of at least 3 years of highly myopic patients and identification of predisposing findings for the development of myopic CNV were examined.

Results: Among 325 highly myopic eyes examined, 33 eyes $(10.2 \%)$ developed myopic CNV. The incidence was higher $(34.8 \%)$ among the fellow eyes of patients with preexisting CNV than among eyes of patients without pre-existing CNV (6.1\%). CNV developed in 3.7\% with diffuse chorioretinal atrophy, in $20.0 \%$ with patchy atrophy, and in $29.4 \%$ with lacquer cracks.

Conclusion: Approximately one in 10 highly myopic eyes developed myopic CNV in average 130.2 months. Patchy atrophy and lacquer cracks were shown to be important predisposing findings for CNV development.
\end{abstract}

$\mathrm{H}$ igh myopia is a major cause of legal blindness in many developed countries. ${ }^{1-3}$ It affects $27 \%$ to $33 \%$ of all myopic eyes, corresponding to a prevalence of $1.7 \%$ to $2 \%$ in the general population of the United States. ${ }^{4}$ In Japan, pathological or high myopia reportedly affects $6 \%$ to $18 \%$ of the myopic population, and around $1 \%$ of the general population. $^{5}$

High myopia is associated with progressive and excessive elongation of the eyeball..$^{6-10}$ As a result of excessive eyeball elongation, various funduscopic changes within the posterior staphyloma develop in highly myopic eyes. ${ }^{11-14}$ Among these myopic fundus lesions, macular choroidal neovascularisation $(\mathrm{CNV})$ is the most common vision threatening complication of high myopia. ${ }^{15-17}$

Despite the importance of CNV as a vision threatening complication, the incidence of myopic CNV has not yet been reported. Some previous reports described the prevalence of myopic CNV. ${ }^{6718}$ For long term management of highly myopic patients, however, it is more important to know how frequently new CNV occurs in myopic eyes. Also, to our knowledge, there have been no studies to investigate the predisposing findings for the development of myopic CNV.

In the present study, many highly myopic patients with myopic fundus changes in the macular lesion were followed, and examined for later development of myopic CNV. The incidence of myopic CNV was identified in these patients as well as possible predisposing findings for the development of myopic CNV.

\section{PATIENTS AND METHODS}

In all, 218 consecutive patients (325 eyes) with high myopia were identified using clinical records from 1988 to 2001 in the high myopia clinic at Tokyo Medical and Dental University and were enrolled in the present study. Informed consent was obtained from all patients. Approval from the ethics committee of the university was obtained. Inclusion criteria for this study were (1) refractive error of $-8 \mathrm{D}$ or more; (2) fundus changes typical of pathological myopia within 1 disc diameter (DD) from the fovea centralis; and (3) minimum follow up period of 3 years. Ages of the patients ranged from 7 to 82 years (mean 48.3 (SD 14.8)). Refractive error ranged from -8.5 to $-36.0 \mathrm{D}$ (mean -16.1 (4.9) D), and axial length ranged from 25.9 to $36.0 \mathrm{~mm}$ (mean 29.8 (1.7) $\mathrm{mm}$ ). Visual acuity ranged from $20 / 200$ to $20 / 20$ (mean $\log$ MAR $0.386(0.354)$ ). Eyes with myopic CNV and patients with a follow up of less than 3 years were excluded from the study. The fellow eyes of patients with pre-existing myopic CNV were included in the study (46 eyes of 46 patients). Additional exclusion criteria included a history of retinal detachment surgery, diabetic retinopathy, or other retinal vascular diseases, glaucoma, and ocular injuries.

Routine ophthalmological examination, direct and indirect binocular ophthalmoscopy, slit lamp biomicroscopy with a contact lens, and fluorescein fundus angiography were performed in all patients. The patients were regularly examined at 6 month intervals. Classification of the posterior fundus changes at the initial examination was independently performed by three authors (KOM, TY, SF) according to Tokoro et al. ${ }^{19}$ Among these fundus changes, diffuse chorioretinal atrophy, lacquer cracks, and patchy chorioretinal atrophy were investigated as possible predisposing lesions for the future development of myopic CNV. Only the fundus changes within 1 DD from the fovea centralis were examined as possible predisposing lesions. Figure 1 is a representative photograph of myopic fundus changes. Briefly, diffuse chorioretinal atrophy (D) appears yellowish-white in the posterior fundus (Fig 1A). In the present study, diffuse atrophy was defined as yellowish fundus without lacquer cracks. We considered lacquer cracks (Fig lB) as a separate category in the present study because a previous report described an important relation between myopic CNV and lacquer cracks. ${ }^{15}$ The ophthalmoscopic finding of patchy atrophy $(\mathrm{P})$ is a greyish-white, well defined lesion (Fig 1C). The development of CNV was confirmed by fluorescein angiography. For the patients who developed CNV during the follow up period, the changes in the myopic fundus that existed in the macula before CNV development were identified as predisposing findings of myopic CNV.

Statistical analyses utilised the $\chi^{2}$ test and Mann-Whitney U test. Probability values smaller than 0.05 are considered to be statistically significant. 

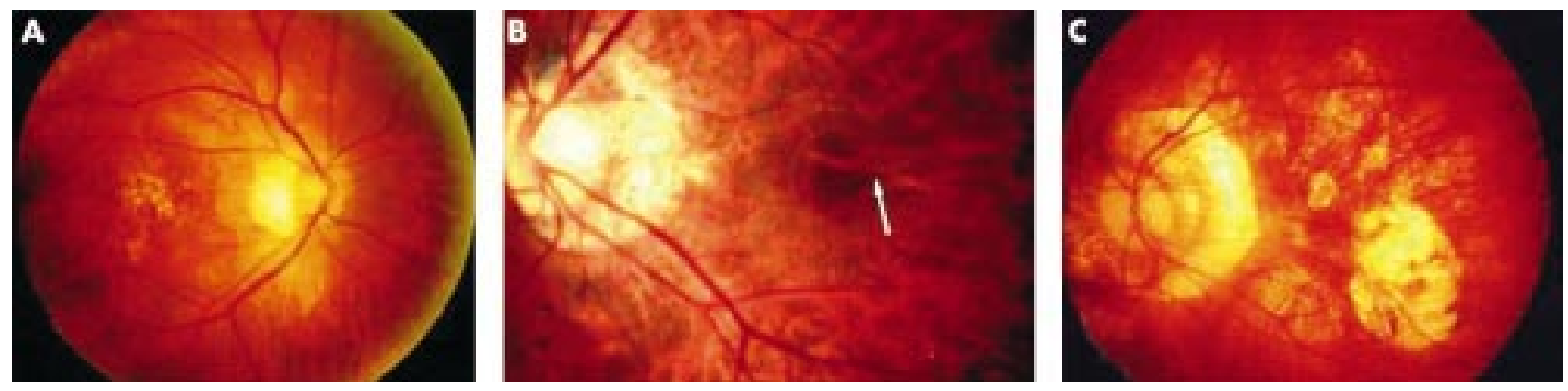

Figure 1 Representative photographs of myopic fundus changes. (A) Diffuse chorioretinal atrophy. A yellowish-white spotty lesion is observed in the macular area. (B) Lacquer cracks. A yellowish linear lesion is observed in the macula (arrow). (C) Patchy atrophy. A greyish-white, well defined lesion of 2 disc diameters is observed in the inferotemporal part of the fovea centralis.

\section{RESULTS}

The follow up period in all patients examined ranged from 36 to 314 months (average 130.2 (69.3) months). Fundus changes at the initial examination were diffuse atrophy in 243 of 325 eyes (74.8\%), patchy atrophy in 40 of 325 eyes (12.3\%), and lacquer cracks in 51 of 325 eyes $(15.7 \%)$. In one eye $(0.3 \%)$, an extremely large optic disc conus reached within IDD from the fovea centralis.

During a follow up period, myopic CNV eventually occurred in 33 of 325 eyes ( 32 patients; 10.2\%). Among these 33 eyes that later developed myopic CNV, CNV occurred in nine eyes with diffuse atrophy, in eight eyes with patchy atrophy, in 15 eyes with lacquer cracks, and in one eye with a large myopic conus. According to each fundus lesion category, CNV developed in nine of 243 eyes (3.7\%) with diffuse atrophy, in eight of 40 eyes $(20.0 \%)$ with patchy atrophy, in 15 of 51 eyes $(29.4 \%)$ with lacquer cracks, and in one of one eye with a large myopic conus.

Among the 33 eyes that developed myopic CNV, 16 eyes $(48.5 \%)$ were the fellow eyes of patients with pre-existing myopic CNV. In other words, among 46 fellow eyes of patients with pre-existing myopic CNV, 16 of 46 eyes (34.8\%) also developed myopic CNV during the follow up. The incidence of $\mathrm{CNV}$ in the fellow eyes of patients with pre-existing myopic $\mathrm{CNV}$ was significantly higher than that in eyes of patients without pre-existing CNV $(34.8 \%$ v $6.1 \%)\left(\mathrm{p}=0.0001, \chi^{2}\right.$ test $)$. There was no significant difference in age, refractive error, axial length, or logMAR between the two groups at the initial visit (data not shown). In 16 fellow eyes of patients with preexisting myopic CNV, CNV developed within 91.7 (52.4) months (range 22-175 months) on average after the occurrence of myopic CNV in the first eye. In 16 fellow eyes of patients with pre-existing myopic CNV, CNV developed in five of 35 eyes $(14.3 \%)$ with diffuse atrophy, in three of four eyes $(75.0 \%)$ with patchy atrophy, and in eight of 15 eyes $(57.1 \%)$ with lacquer cracks.

Representative cases are shown in Figures 2 and 3.

\section{DISCUSSION}

To our knowledge, this is the first report describing the predisposing findings for and the precise incidence of myopic CNV in a large series of highly myopic patients. In the present study, over a follow up period of at least 3 years, myopic CNV developed at a rate of $10.2 \%$ (33 of 325 eyes), demonstrating that approximately $1 / 10$ of highly myopic eyes with myopic fundus changes in the macula can develop myopic CNV within 3 or more years.

Also in the present study, 46 of 325 eyes were actually the fellow eyes of patients with pre-existing myopic CNV. The result indicated a higher incidence of CNV development in the
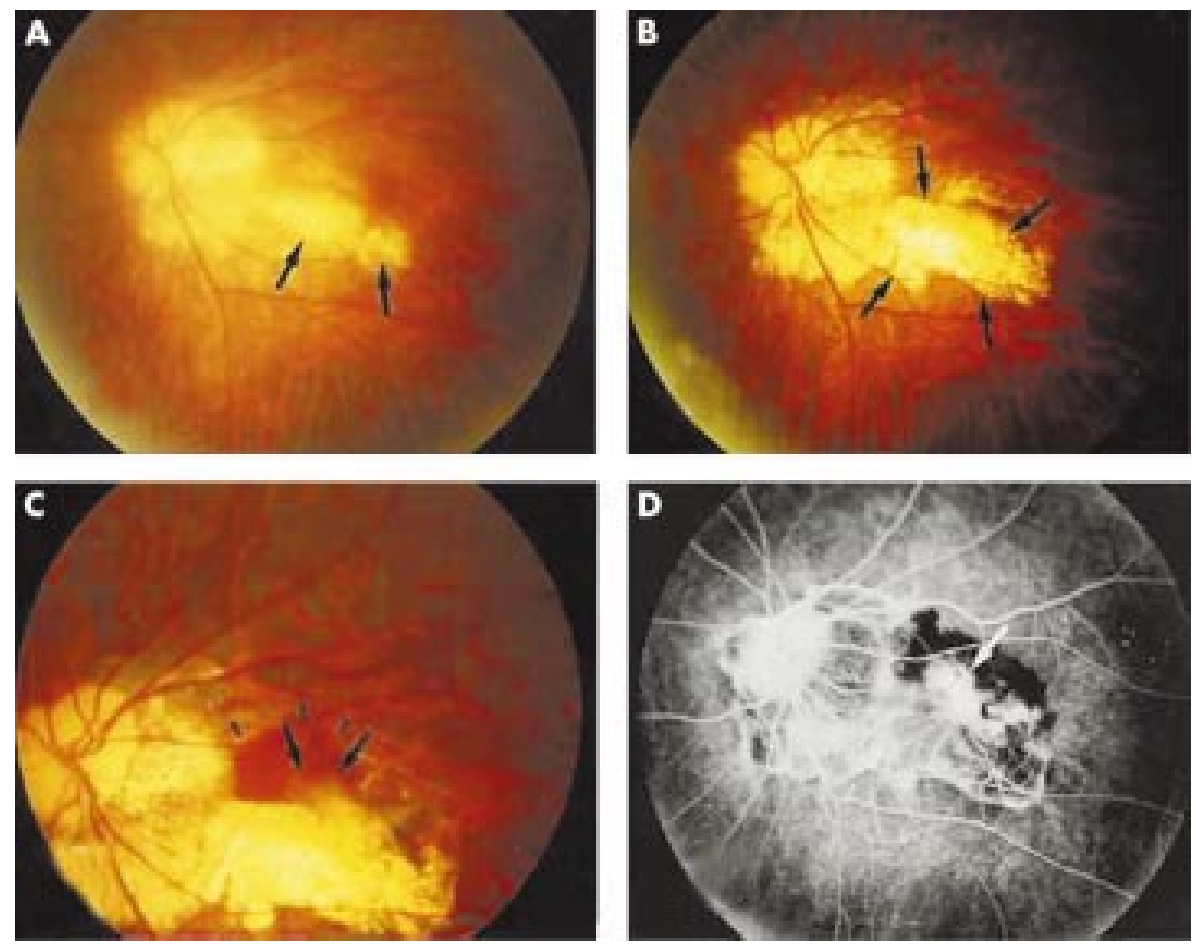

Figure 2 Development of myopic CNV at the edge of the area of patchy atrophy. Patient 1. A 47 year old woman. (A) Left fundus at the initial examination (January 1991) showed an area of patchy atrophy below the macula (arrows). (B) Two years later (August 1993), the area of patchy atrophy enlarged and coalesced (arrows). (C) One year later (June 1994), CNV developed at the macular edge of the area of patchy atrophy (arrows) Subretinal bleeding was observed around the CNV (arrowheads). (D) Fluorescein angiogram at the onset of CNV. At 2 minutes after dye injection, there was intense hyperfluorescence corresponding to the site of the CNV (white arrow). The patient's visual acuity dropped from $20 / 25$ to $20 / 70$. 

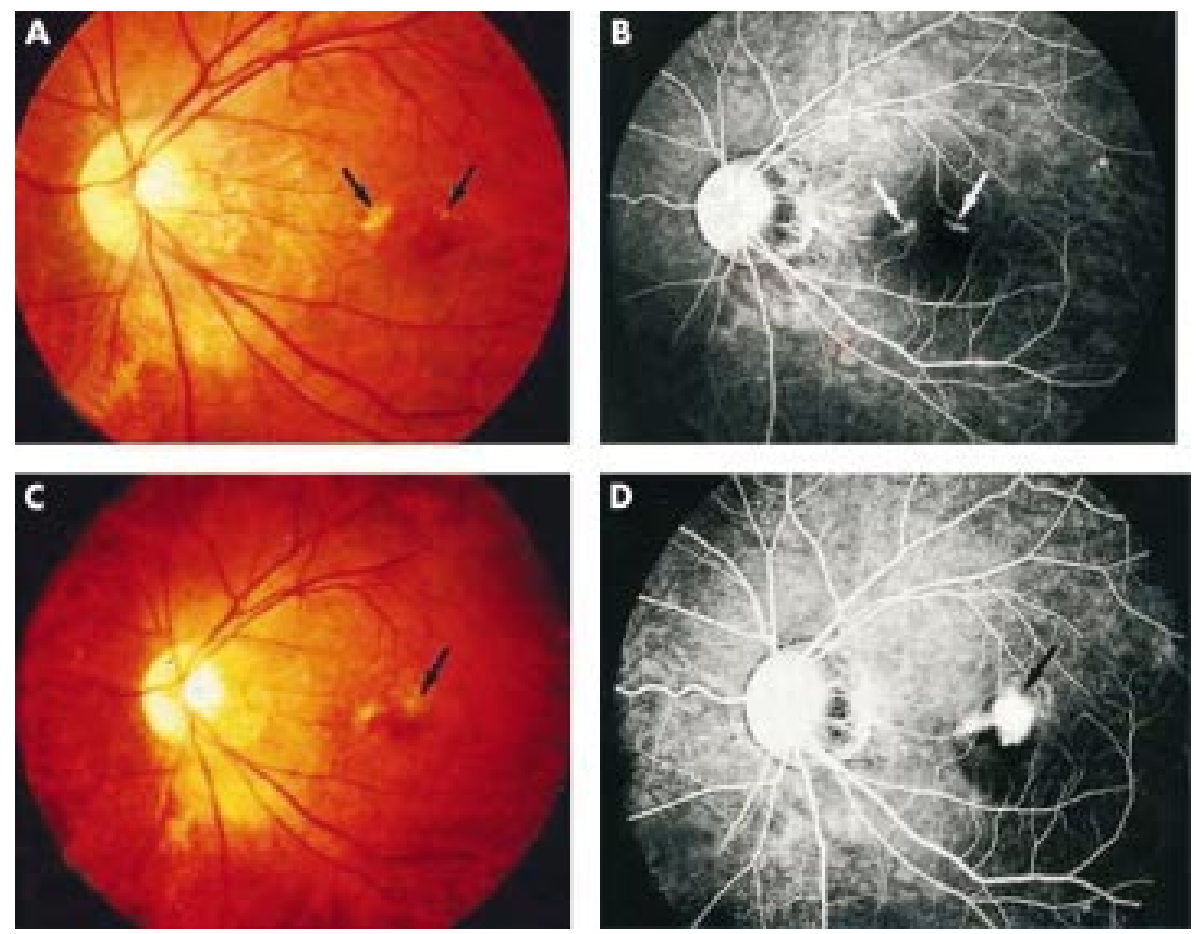

Figure 3 Development of myopic CNV from lacquer cracks. Patient 3 A 28 year old woman. (A) Left fundus at the initial examination (November 1993) revealed two lacquer cracks above the macula (arrows). (B) Fluorescein angiogram at the initial examination showed linear hyperfluorescence corresponding to the lacquer cracks (arrows). (C) Three years later (December 1996), CNV developed at corresponding site of previous lacquer crack (arrow). (D)

Fluorescein angiogram at the onset of CNV. At 1 minute after dye injection, there was intense hyperfluorescence corresponding to the site of the CNV (arrow). The patient's visual acuity dropped from $20 / 20$ to $20 / 200$. fellow eyes of patients with pre-existing CNV (34.8\%) than eyes of patients without pre-existing CNV (6.1\%). The mean period until the development of CNV in the second eye after the development of CNV in the first eye was relatively long, 91.7 (52.4) months. Thus, this study indicates that CNV occurs in the fellow eyes of patients with pre-existing myopic CNV at a high rate (more than $30 \%$ ) within approximately 8 years after CNV development in the first eye. Therefore, a careful and long term follow up is recommended, especially for the fellow eyes of patients with pre-existing myopic CNV.

The present study demonstrated that among various myopic fundus changes, patchy atrophy and lacquer cracks are especially important predisposing lesions. Twenty per cent of eyes with patchy atrophy within IDD from the fovea centralis and $29.4 \%$ of eyes with lacquer cracks developed myopic CNV during the follow up period. Although lacquer cracks have been the only lesion regarded to have an important relation with myopic $\mathrm{CNV}^{15}$ no reports have demonstrated the development of CNV at the site of the lacquer cracks. Lacquer cracks are considered to represent mechanical fissures in the retinal pigment epithelium-Bruch's membranechoriocapillaris complex secondary to eyeball elongation in highly myopic eyes. ${ }^{12}$ Thus, the present study suggests that a wound healing mechanism might underlie the development of CNV in some myopic patients.

Patchy atrophy is the second most common predisposing finding for the development of CNV. Patchy atrophy is considered to represent complete atrophy of the retinal pigment epithelium and choriocapillaris. ${ }^{19}$ Although the mechanism of the development of CNV in eyes with patchy atrophy is unclear, it is likely that retinal pigment epithelium and Bruch's membrane are mechanically damaged at the edge of these lesions, and this might result in later development of CNV.

In summary, approximately $10 \%$ of highly myopic eyes with myopic fundus lesions within IDD from the fovea develop myopic CNV within an average of 130.2 months. In particular, eyes with patchy atrophy or lacquer cracks around the macula have a higher risk of developing myopic CNV (around 20\%). Also, in the fellow eyes of patients with pre-existing myopic $\mathrm{CNV}$, the incidence of developing $\mathrm{CNV}$ in the second eye is higher than that in the eyes of patients without pre-existing CNV. Almost $30 \%$ of the fellow eyes of patients with pre-existing myopic CNV eventually develop CNV within an average of 8 years. These findings might be very beneficial for the clinical management of high risk patients.

\section{ACKNOWLEDGEMENT}

Supported in part by research grant 12671698 from the Japanese Ministry of Education, Culture, Sports, Science and Technology.

\section{Authors' affiliations}

K Ohno-Matsui, T Yoshida, S Futagami, K Yasuzumi, N Shimada, A Kojima, T Tokoro, M Mochizuki, Department of Ophthalmology and Visual Science, Tokyo Medical and Dental University, Tokyo, Japan

Correspondence to: Kyoko Ohno-Matsui, MD, Department of Ophthalmology and Visual Science, Tokyo Medical and Dental University, 1-5-45 Yushima, Bunkyo-ku, Tokyo 113, Japan; k.ohno.oph@med.tmd.ac.jp

Accepted for publication 26 Septemver 2002

\section{REFERENCES}

1 Ghafour IM, Allan D, Foulds WS. Common causes of blindness and visual handicap in the West of Scotland. Br J Ophthalmo 1983;67:209-13.

2 Tokoro T. Criteria for diagnosis of pathologic myopia. In: Tokoro T, ed. Atlas of posterior fundus changes in pathologic myopia. Tokyo: Springer Verlag, 1998: 1-2

3 Sperduto RD, Seigel D, Roberts J, et al. Prevalence of myopia in the United States. Arch Ophthalmol 1983;101:405-7.

4 Curtin BJ. The nature of pathologic myopia. In: Curtin BJ, ed. The myopias. Philadelphia: Harper and Row, 1985:237-45.

5 Tokoro T. On the definition of pathologic myopia in group studies. Acta Ophthalmol 1988;66:107-8.

6 Curtin BJ, Karlin DB. Axial length measurements and fundus changes of the myopic eye. Am J Ophthalmol 1971;71:42-50.

7 Grossniklaus HE, Green WR. Pathologic findings in pathologic myopia. Retina 1992;12:127-33

8 Curtin BJ. Physiologic vs pathologic myopia: genetics vs environment. Ophthalmology 1979;86:681-91.

9 Gass JDM. Myopic choroidal degeneration. In: Gass JDM, ed. Stereoscopic atlas of macular diseases: diagnosis and treatment. 3rd ed. Vol 1. St Louis: Mosby, 1997:110-13.

10 Curtin BJ. Posterior staphyloma development in pathologic myopia. Ann Ophthalmol 1982;14:655-8

11 Steidl SM, Pruett RC. Macular complications associated with posterior staphyloma. Am J Ophthalmol 1997;123:181-7. 
12 Curtin BJ. Ocular findings and complications. In: Curtin BJ, ed. The myopias. Philadelphia: Harper and Row 1985:277-347.

13 Rabb MF, Garoon I, LaFranco FP. Myopic macular degeneration. Int Ophthalmol Clin 1981;21:51-69.

14 Noble KG, Carr RE. Pathologic myopia. Ophthalmology 1982;89:1099-100.

15 Avila MP, Weiter JJ, Jalkh AE, et al. Natural history of choroidal neovascularization in degenerative myopia. Ophthalmology 1984;91:1573-81.
16 Hotchkiss ML, Fine SL. Pathologic myopia and choroidal neovascularization. Am J Ophthalmol 1981;91:177-83.

17 Hampton GR, Kohen D, Bird AC. Visual prognosis of disciform degeneration in myopia. Ophthalmology 1983;90:923-6.

18 Levy JH, Pollock HM, Curtin BJ. The Fuchs' spot. An ophthalmoscopic and fluorescein angiographic study. Ann Ophthalmol 1977;9:1433-42

19 Tokoro T. Types of fundus changes in the posterior pole. In: Tokoro T, ed. Atlas of posterior fundus changes in pathologic myopia. Tokyo: Springer-Verlag 1998:5-22.

Some patients rate risk of complications not worth the wait for cataract operations

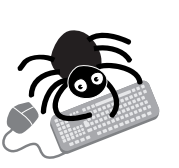

Please visit the British Journal of Ophthalmology website [www. bjophthalmol.com for link to this full article.
Ome older patients are theoretically willing to trade a higher risk of complications

against shorter waiting times for cataract operations, a survey has disclosed. In a radical proposal, the finding might permit patients a choice between risk and waiting time by opting for a junior or consultant surgeon, say the investigators.

The interview survey of a sample of the general public in the UK aged 60-84 showed that the 146 respondents rated the risk of complications and waiting time as more important than surgeon grade (median importance score $46 \%, 41 \%$ v 13\%, respectively). However, analysing the non-normally distributed responses on an individual basis showed that for some respondents risk of complications was more important than waiting time-and vice versa The sample was typical of the general UK population for age, sex, cataract, and Jarman deprivation scores. The characteristics of the responders and non-responders were similar. Only length of education and being a driver or doing some other visually exacting tasks influenced preferences-making risk of complication more important.

The interviewees were systematically selected from GP registers in one health authority, those with difficulties in communicating or aged over 84 being excluded. They were presented with an array of 11 theoretical options around permutations of waiting time $(4,8$, and 16 months), complication rate $(1 \%, 5 \%, ! 0 \%)$, and surgeon grade (junior/consultant), which they had to choose between in order of preference. The data were analysed by conjoint analysis.

ه Quality and Safety in Health Care 2003;23:13-17. 